\title{
Incidência e Mortalidade por Câncer entre Crianças e Adolescentes: uma Revisão Narrativa
}

doi: https://doi.org/10.32635/2176-9745.RBC.2018v64n3.45

Cancer Incidence and Mortality among Children and Adolescents: a Narrative Review
Incidencia y Mortalidad por Cáncer entre Niños e Adolescentes: una Revisión Narrativa

Suellen Valadares Moura Feliciano'; Marceli de Oliveira Santos²; Maria S. Pombo-de-Oliveira ${ }^{3}$

Resumo

Introduçáo: $\mathrm{O}$ câncer em crianças e adolescentes com idade entre 0-19 anos configura-se como um problema de saúde pública tanto nos países desenvolvidos como nos países em desenvolvimento. É considerado raro e distinto quando comparado com o câncer em adultos. Apresenta padróes de incidência e mortalidade que variam em todo mundo, sendo que aproximadamente $80 \%$ dos cânceres infantis ocorrem nos países com baixo índice de desenvolvimento humano, com acesso aos serviços de cuidado à saúde de baixa qualidade. Objetivo: Apresentar um panorama geral do câncer em crianças e adolescentes com idade entre 0-19 anos com ênfase na descriçáo dos resultados de estudos de base populacional para incidência e mortalidade nas diferentes regióes geográficas no Mundo e no Brasil. Resultados: As taxas de incidência gerais para o câncer em crianças e adolescentes com idade entre 0-19 variaram entre 50 e 200 casos por milhão por ano em diferentes países e continentes. No Brasil, a mediana das taxas a incidência foi de 154,3 por milhão. A mortalidade apresentou declínio em várias partes do mundo, sendo considerada a segunda causa de morte em países desenvolvidos. Conclusáo: As informaçóes dos registros de câncer são indispensáveis no enfrentamento do câncer na populaçáo pediátrica, principalmente nos países em desenvolvimento, onde o impacto do câncer é pouco conhecido, ao passo que seu efeito sobre a população está aumentando. Melhorias na adoção de estratégias de tratamento integrado devem ser consideradas para melhorar as taxas de mortalidade por câncer em crianças e adolescentes no Brasil e no Mundo.

Palavras-chave: Adolescente; Criança; Incidência; Mortalidade; Neoplasias; Registros Eletrônicos de Saúde.

\section{Abstract}

Introduction: Cancer in children and adolescents aged 0-19 years is a public health problem in both developed and developing countries. It is considered rare and distinct when compared to cancer in adults. They present patterns of incidence and mortality vary throughout the world, with approximately $80 \%$ of childhood cancers occurring in low human development countries, with access to poor quality health care services. Objective: To present an overview of cancer in children and adolescents aged 0-19 years, with an emphasis on describing the results of population-based studies for incidence and mortality in different geographic regions in the World and Brazil. Results: Overall cancer incidence rates in children and adolescents aged $0-19$ years old ranged from 50 to 200 cases per million per year in different countries and continents. In Brazil, the median incidence rates were 154.3 per million. Mortality declined in several parts of the world, being considered the second cause of death in developed countries. Conclusion: The information from the cancer registries are indispensable in coping with cancer in the pediatric population, especially in developing countries, where the impact of cancer is poorly understood, while its effect on the population is increasing. Improvements in the adoption of integrated treatment strategies should be considered to improve cancer mortality rates in children and adolescents in Brazil and in the World.

Key words: Adolescent; Child; Incidence; Mortality; Neoplasms; Electronic Health Records.
Resumen

Introducción: El cáncer en niños y adolescentes de entre 0-19 años se configura como un problema de salud pública tanto en los países desarrollados y en los países en desarrollo. Se considera raro y distinto cuando se compara con el cáncer en adultos. Se presentan patrones de incidencia y mortalidad que varían en todo el mundo, siendo que aproximadamente el $80 \%$ de los cánceres infantiles ocurren en los países con bajo índice de desarrollo humano, con acceso a los servicios de cuidado de la salud de baja calidad. Objetivo: Presentar un panorama general del cáncer en nińos y adolescentes con edad entre 0-19 ańos con énfasis en la descripción de los resultados de estudios de base poblacional para incidência y mortalidad en las diferentes regiones geográficas en el Mundo y en Brasil. Resultados: Las tasas de incidencia general para el cáncer en niños y adolescentes de entre 0-19 varían entre 50 y 200 casos por millón por año en diferentes países y continentes. En Brasil, la mediana de las tasas la incidencia fue de 154,3 por millón. La mortalidad ha disminuido en varias partes del mundo, siendo considerada la segunda causa de muerte en los países desarrollados. Conclusión: La información de los registros de cáncer es indispensable en el enfrentamiento del cáncer en la población pediátrica, principalmente en los países en desarrollo, donde el impacto del cáncer es poco conocido, mientras que su efecto sobre la población está aumentando. Las mejoras en la adopción de estrategias de tratamiento integrado deben ser consideradas para mejorar las tasas de mortalidad por cáncer en nińos y adolescentes en Brasil y en el Mundo.

Palabras clave: Adolescente; Nińo; Incidencia; Mortalidad; Neoplasias; Registros Electrónicos de Salud.

\footnotetext{
1 Instituto Nacional de Câncer José Alencar Gomes da Silva (INCA). Rio de Janeiro (RJ), Brasil. Orcid iD: https://orcid.org/0000-0002-2762-3414

${ }^{2}$ INCA. Rio de Janeiro (RJ), Brasil. Orcid iD: https://orcid.org/0000-0001-5197-2019

${ }^{3}$ INCA. Rio de Janeiro (RJ), Brasil. Orcid iD: http://orcid.org/0000-0003-3986-8993

Endereço para correspondência: Suellen Valadares Moura Feliciano. Praça Cruz Vermelha, 23, 6 andar - Centro. Rio de Janeiro (RJ), Brasil. CEP 20230-130. E-mail: suellen.moura@inca.gov.br.
} 


\section{INTRODUÇÃO}

O câncer infantojuvenil, definido como câncer em crianças e adolescentes com idade entre 0-19 $\operatorname{anos}^{1-3}$, configura-se como um problema de saúde pública tanto nos países desenvolvidos como nos países em desenvolvimento. É considerado raro quando comparado com o câncer em adultos, correspondendo a apenas uma pequena proporção da carga global do câncer, com frequência de incidência média estimada entre $0,5 \%$ a $4,6 \%$ de todos os tumores malignos ${ }^{4-6}$, sendo que aproximadamente $80 \%$ dos cânceres infantis ocorrem em países com baixo índice de desenvolvimento humano (IDH), com acesso aos serviços de cuidado à saúde de baixa qualidade ${ }^{6,7}$.

Os cânceres infantis são entidades distintas dos cânceres que ocorrem em adultos, e devem ser estudados separadamente em razão das diferenças nos sítios primários, origens histológicas e comportamentos clínicos $^{8}$. Enquanto o câncer no adulto está associado à açáo de vários fatores de risco como tabagismo, dieta, ocupação e exposição a outros agentes carcinogênicos, as associaçōes causais para o câncer infantil ainda são pouco exploradas e os possíveis mecanismos envolvidos nesse processo permanecem desconhecidos ${ }^{4-5}$. Estudos sugerem associação com a predisposição genética, hereditária, imunológica, exposição ambiental a agentes genotóxicos, radiaçôes ionizantes, campos eletromagnéticos e outros $^{9-11}$.

Investigaçóes que permitam avaliar características e diferenças epidemiológicas em países desenvolvidos e em desenvolvimento são insubstituíveis, uma vez que essas populaçôes estão expostas a diferentes ambientes, condiçôes socioeconômicas e fatores de $\operatorname{risco}^{12}$. A identificação dessas diferenças geográficas, especialmente em populaçóes geneticamente relacionadas, pode fornecer subsídios sobre as possíveis associaçôes etiológicas, e tem sido utilizada para apoiar hipóteses da associação entre as exposiçóes relacionadas ao estilo de vida e o risco de câncer infantil, particularmente a leucemia ${ }^{13}$.

As informaçôes dos registros de câncer de base populacional (RCBP) sobre incidência e mortalidade contribuem não apenas para o entendimento da etiologia da doença, mas também para monitorar as mudanças no impacto da doença, a qualidade da assistência prestada e o tratamento de cânceres infantis ${ }^{12}$.

Diante do exposto, esta revisão objetiva apresentar um panorama geral do câncer em crianças e adolescentes com idade entre 0-19 anos, com ênfase na descriçáo dos resultados de estudos de base populacional para incidência e mortalidade nas diferentes Regiôes Geográficas no Mundo e no Brasil.

\section{CLASSIFICAÇÃO DO CÂNCER EM CRIANÇAS E ADOLESCENTES}

O câncer em crianças (0-14 anos) possui uma classificação própria, a Classificação Internacional do Câncer na Infância, terceira edição (CICI-3). Baseada na morfologia ao invés do local primário de origem do tumor, como é feita nos adultos, essa classificação foi desenhada para fins epidemiológicos internacionais e uniformização das informaçóes dos registros de base populacional, o que permite a comparaçáo das informaçôes de incidência e sobrevida em diferentes regióes e períodos. Ressalta-se que o uso de um sistema de classificação internacional é especialmente importante no campo da pediatria oncológica, em que a baixa frequência de casos requer procedimentos rigorosos para assegurar a comparabilidade das casuísticas ${ }^{14}$.

A CICI-3 divide o câncer em 12 grupos principais, que se subdividem em 47 subgrupos: I. leucemias, doenças mieloproliferativas e mielodisplásicas; II. linfomas e neoplasias reticuloendoteliais; III. tumores do sistema nervoso central (SNC) e miscelânea de neoplasias intracranianas e intraespinhais; IV. tumores do sistema nervoso simpático; V. retinoblastoma; VI. tumores renais; VII. tumores hepáticos; VIII. tumores ósseos malignos; IX. sarcomas de partes moles; X. neoplasias de células germinativas, trofoblásticas e outras gonadais; XI. carcinomas e outras neoplasias malignas epiteliais; XII. outros tumores malignos não especificados ${ }^{14}$.

O câncer em adolescentes e adultos jovens (1524 anos) apresenta um padrão diferenciado quando comparado às crianças e aos adultos. Em 2002, Birch et al. ${ }^{15}$ propuseram uma classificação específica para essa faixa etária, cuja versão brasileira é denominada como Esquema de Classificação para Tumores em Adolescentes e Adultos Jovens (CAAJ), sendo incorporada aos RCBP desde 2015.

\section{EPIDEMIOLOGIA DO CÂNCER EM CRIANÇAS E ADOLESCENTES}

Estudos têm demonstrado que as taxas de incidência do câncer em crianças e adolescentes apresentam diferentes padróes geográficos e vêm aumentando ao longo do tempo em todas as faixas etárias. Cerca de $1 \%$ de aumento ao ano tem sido observado nas últimas três décadas em Regiōes como Europa, América do Norte, Austrália e outros lugares, embora a taxa de aumento pareça ter estabilizado na década mais recente ${ }^{16-19}$.

Os RCBP em todo o mundo relatam taxas de incidência gerais para o câncer infantil que variam entre 50 e 200 casos por milhão de crianças por ano em diferentes países. Sendo que o espectro dos tipos de tumor difere entre as populaçóes ${ }^{17,20-29}$, com tumores infantis particularmente raros, com taxas de incidência que variam de 1 por milhão, como o hepatoblastoma, a 50 por milhão para o subgrupo mais comum, a leucemia linfoide aguda (LLA) ${ }^{2}$. 
A explicação para as diferenças na incidência total em todo o mundo é amplamente desconhecida, embora provavelmente esteja relacionada às diferenças na exposição a fatores de risco, juntamente com fatores genéticos que afetam a probabilidade de o indivíduo desenvolver o câncer ${ }^{30}$. Na África equatorial, por exemplo, o linfoma de Burkitt representa cerca de $50 \%$ de todos os cânceres de infância, e a epidemia de Aids em muitos países africanos levou ao aumento substancial do sarcoma de Kaposi, que apresenta incidência maior do que o linfoma de Burkitt nessas regiōes ${ }^{30-32}$. Além disso, alguns tipos de câncer podem variar de acordo com a extensão do desenvolvimento socioeconômico, como por exemplo a LLA, muito comum principalmente em países com populaçôes com alto nível socioeconômico. Uma das evidências para essa sugestão é fornecida pela queda da incidência do linfoma de Burkitt e pelo aumento de sua incidência na faixa de Gaza que coincidiu com um período de rápido desenvolvimento socioeconômico ${ }^{33}$. Outro câncer relacionado com o desenvolvimento econômico é retinoblastoma não hereditário que tem maior incidência entre as populaçóes menos desenvolvidas, o que sugere uma associação com baixa condição socioeconômica e, talvez, etiologia infecciosa. Em contraste, a incidência do tumor de Wilms e do sarcoma de Ewing varia amplamente em linhas étnicas, indicando um papel importante para a predisposição genética. Esses tumores podem ser originários de células embrionárias que persistem após o nascimento ou de eventos oncogênicos ocorridos intraútero ${ }^{1,4,30-36}$.

\section{INCIDÊNCIA POR CÂNCER EM CRIANÇAS E ADOLESCENTES NO MUNDO}

Com base nas estimativas do Globocan 2012, a incidência do câncer em crianças com idade entre 0-14 anos para todos os tipos de câncer, exceto pele não melanoma, em todo o mundo, foi de 88 por milhão, sendo 98 por milhão para o sexo masculino e 76 por milhão para o sexo feminino. Nas regióes mais desenvolvidas, foi de 145 por milhão e, nas menos desenvolvidas, foi de 81 por milhão. Na América do Norte (Canadá e Estados Unidos da América), foi de 165 por milhão, na Europa 139 por milhão, na Oceania 120 por milhão, na América Latina e Caribe de 109 por milhão, na África 85 por milhão, e na Ásia foi 75 por milhão .

$\mathrm{Na}$ Europa, um estudo, originado a partir das informaçóes do Automated Childhood Cancer Information System (ACCIS), analisou a tendência por câncer na criança e no adolescente. Nesse estudo, foram analisados 62 RCBP localizados em 19 países europeus, totalizando 113 mil casos novos de câncer em crianças (0-14 anos) e 18 mil em adolescentes (15-19 anos) no período de 1970 a 1999. Foi observado que, na década de 1990 , as taxas de incidência ajustadas por idade eram 140 por milhão em crianças e 157 por milhão em crianças e adolescentes (0-19 anos). Ao longo das três décadas, a incidência global cresceu $1,0 \%$ ao ano em crianças, com aumento para a maioria dos tipos de tumor, e 1,5\% em adolescentes ${ }^{1}$.

Em relação à distribuição dos tumores por faixa etária, foi observado que o neuroblastoma (28\%) foi o tipo de tumor mais frequente em lactentes com idade menor que 1 ano. Em crianças com idade entre 1-4 anos, foram as leucemias (41\%) e, no grupo de 5-9 anos, os tumores do SNC (28\%). Após 10 anos de idade, os tumores embrionários, como o retinoblastoma, nefroblastoma e hepatoblastoma quase desapareceram, enquanto outros cânceres se tornaram mais frequentes, principalmente os linfomas, carcinomas, tumores de células germinativas e tumores ósseos. Nos adolescentes, os linfomas representaram $25 \%$ e os carcinomas $20 \%$ do total. Em relação à incidência, no geral, os tipos de tumores mais incidentes em crianças foram as leucemias com 44,8 por milhão, tumores SNC (29,8 por milhão) e linfomas (15,5 por milhão). Em adolescentes, as maiores taxas de incidência foram para os linfomas (47,4 por milhão), seguidas por carcinomas ( 38,1 por milhão), tumores do SNC (24,6 por milhão), tumores de células germinativas $(24,5 \text { por milhão), e leucemias (23,4 por milhão })^{1}$.

Recentemente, Steliarova-foucher et al..$^{37}$ analisaram uma década (2001-2010) de informação de 153 registros localizados em 62 países, departamentos e territórios. A taxa de incidência ajustada por idade global foi de 140,6 por milhão em crianças ( $0-14$ anos) e 155,8 por milhão com idade entre 0-19 anos. E os cânceres mais comuns foram leucemia (46,4 por milhão), seguidos por tumores do SNC (28,2 por milhão) e linfomas (15,2 por milhão). Em adolescentes com idade entre de 15-19 anos, a taxa de incidência específica por idade foi de 185,3 por milhão, sendo os mais comuns os linfomas ( 41,8 por milhão) e o grupo de tumores epiteliais e melanoma (39,5 por milhão). Foi observado também que a incidência variou consideravelmente por regiốes, tipo de câncer, sexo, idade e grupo étnico. Além disso, desde a década de 1980, o índice global de cânceres registrados em crianças de 0-14 anos aumentou de 124,0 para 140,6 por milhão.

Nos Estados Unidos, dois estudos realizados a partir das informaçóes do Surveillance, Epidemiology, and End Results (SEER) program analisaram a incidência por câncer em crianças e adolescentes. Siegel et al. ${ }^{38}$ observaram que a taxa de incidência ajustada por idade foi de 171,01 por milháo durante o período de 2001-2009. Sendo que a taxa global para todos os cânceres combinados permaneceu estável ao longo do tempo, com aumento na tendência geral em afro-americanos com idade entre 
0-19 anos (variaçãoo percentual anual, APC:1,3\% [IC95\%: $0,2 ; 2,5]$ ), e, para o câncer de tireoide, em ambos os sexos (APC: 4,9\% [IC 95\%: 3,2;6,6]) e especificamente entre adolescentes e nas Regióes Nordeste, Sul e Oeste dos Estados Unidos, e carcinoma renal (APC: 5,4\% [IC 95\%: 2,8;8,1]). Os tumores de células germinativas extracranianas e extragonadais e melanoma apresentaram diminuição das taxas. Burkhamer, Kriebel e Clapp ${ }^{39}$ observaram que a incidência durante o período de 1975 a 2012 aumentou anualmente em $0,67 \%$ para homens e $0,62 \%$ para mulheres, resultando em um aumento de mais de 25\% em 38 anos. Os maiores aumentos anuais na incidência ocorreram para linfoma não Hodgkin (LNH) (2,16\% em mulheres; $1,38 \%$ em homens), câncer de tireoide (2,12\% em mulheres; $1,59 \%$ em homens), leucemia mieloide aguda (LMA) (1,73\% em mulheres) e câncer de testículo (1,55\% em homens).

\section{INCIDÊNCIA POR CÂNCER EM CRIANÇAS E ADOLESCENTES NO BRASIL}

No Brasil, conforme observado nos RCBP, o percentual mediano dos tumores na população infantil (0-14 anos) foi de 2\%; enquanto, em crianças e adolescentes (0-19 anos), foi de 3\% e, em adolescentes e adultos jovens (15-29 anos), de 4,3\%. Entre as neoplasias, a leucemia foi o tipo de câncer mais incidente com 33\% para os casos com idade entre 0-14 anos e 26\% para 0-19 anos. No grupo etário de 0-14 anos, observou-se que os tumores do SNC se apresentaram como o segundo tumor mais incidente com $16 \%$, seguidos dos linfomas com $14 \%$; enquanto, para o grupo de 0-19 anos, os linfomas apresentaram-se como o segundo tumor mais incidente, seguidos dos tumores do SNC, com $14 \%$ e $13 \%$, respectivamente. Na população de adolescentes e adultos jovens, foi observado um padrão diferenciado quando comparado por outras faixas etárias. O grupo dos carcinomas foi o mais frequente com $34 \%$, seguido dos linfomas (12\%) e dos tumores de pele, incluindo melanomas e carcinomas (9\%). As leucemias nessa faixa etária representaram $8 \%$ de todas as neoplasias, com mediana das taxas de incidência de 21,21 por milhão em ambos os sexos. Em relação à incidência, para todas as neoplasias, observou-se taxa de incidência ajustada por idade global de 126,65 por milháo em crianças com idade entre 0-14 anos; 139 ,99 por milhão (0-19 anos); e 236,16 por milhão (15-29 anos $)^{40}$.

$\mathrm{Na}$ última década, três estudos brasileiros analisaram as informaçóes sobre incidência do câncer infantil ${ }^{41-43}$. De Camargo et al. ${ }^{41}$ avaliaram a informação da incidência do câncer infantil em 14 RCBP (correspondendo a $15 \%$ da população de crianças e adolescentes do Brasil) e mostraram que a mediana das taxas foi de 154,3 por milhão de crianças e adolescentes com idade entre 0-19 anos. As crianças com idade entre 1-4 anos foram as que apresentaram as maiores taxas de incidência. Em todos os RCBP analisados, a leucemia foi o tumor mais incidente, com uma taxa variando de 67,5 por milhão em Goiânia a 21,0 por milhão em Salvador. Seguido de linfoma e tumores de SNC para a maioria dos RCBP estudados. De Camargo et al. ${ }^{42}$ analisaram as taxas de incidência do câncer infantil de acordo com os níveis socioeconômicos (Social Economic Status - SES) das regióes estudadas para os tumores embrionários, como retinoblastoma, neuroblastoma, tumor de Wilms e meduloblastoma. Foram observadas variaçóes regionais diretamente associadas com o SES principalmente para as taxas de neuroblastoma e retinoblastoma. Ferreira et al. ${ }^{43}$ analisaram as taxas de incidência de linfomas em crianças e adolescentes por índices de exclusão social (Social Exclusion Index - SEI) como sendo uma variável de aproximação do nível socioeconômico. Foram observados que os maiores SEI tinham correlaçáo com maior incidência de linfoma de Hodgkin $(\mathrm{LH})(p=0,06)$, embora náo significante.

\section{MORTALIDADE POR CÂNCER EM CRIANÇAS E ADOLESCENTES}

As taxas de mortalidade em crianças e adolescentes com câncer também têm apresentado diferenças nos padrôes geográficos, bem como declínio em várias partes do mundo ${ }^{44-45}$. Em países desenvolvidos, a neoplasia é considerada a segunda causa de morte em crianças com idade entre 0-14 anos, com aproximadamente 5\% dos óbitos nessa faixa etária. Nos países em desenvolvimento, essa proporção é menor (aproximadamente 1\%), em razão das mortes causadas por doenças infecciosas ${ }^{2}$.

\section{MORTALIDADE POR CÂNCER EM CRIANÇAS E ADOLESCENTES NO MUNDO}

Com base nas estimativas do Globocan 2012, a mortalidade por câncer em crianças para todos os tipos de câncer, exceto pele náo melanoma, em todo o mundo, foi de 43 por milhão, sendo 48 por milhão para o sexo masculino e 38 por milhão para o sexo feminino. Nas regiôes menos desenvolvidas, foi de 45 por milhão e, nas mais desenvolvidas, de 26 por milhão. Na África, foi 50 por milhão; na América Latina e Caribe, de 45 por milhão; na Ásia, 43 por milhão; na Oceania, 31 por milhão; na Europa, 29 por milhão; e, na América do Norte (Canadá e Estados Unidos da América), 23 por milhão.

Na Europa, Bosetti et al. ${ }^{44}$ observaram, por meio de informaçóes da Organização Mundial da Saúde (OMS), que a mortalidade para todas as neoplasias infantis diminuiu de forma constante no período entre 1990-2007 (de 52 para 35 por milháo de meninos, e de 43 para 28 por milhão de meninas). Entretanto, foram 
observadas diferenças nos padróes de mortalidade de acordo com as regióes geográficas. Em 2005-2007, as taxas de mortalidade por câncer infantil foram maiores nos países localizados no Leste Europeu (49 e 39 por milhão de meninos e meninas, respectivamente) e Sul ( 40 e 31 por milhão de meninos e meninas, respectivamente) quando comparado com a Europa Ocidental (31 e 25 por milhão de meninos e meninas, respectivamente) e o Norte Europeu (32 e 25 por milhão meninos e meninas, respectivamente).

Chatenoud et al. ${ }^{45}$, com o objetivo de avaliar os padrôes de mortalidade por câncer infantil em várias áreas do mundo, analisaram as informações mortalidade da OMS de 24 países na América, Ásia e Oceania, entre o período de 1970-2007. Nessa análise, observou-se que, desde 1970, a mortalidade para todos os cânceres infantis diminuiu de aproximadamente 80 para 30 por milháo de meninos, e de 60 para 20 por milhão de meninas na América do Norte e no Japão. Os países latino-americanos registraram taxas de aproximadamente 50 e 40 por milháo de meninos e meninas, respectivamente, entre 20052007, e foram semelhantes às taxas registradas em áreas mais desenvolvidas no início dos anos 1980. Além disso, as análises dos padróes geográficos e tendência temporal nos diversos países demonstraram que, nos últimos 35 anos, a mortalidade do câncer infantil reduziu em aproximadamente $60 \%$ em países como Estados Unidos, Canadá e Porto Rico, e correspondeu à prevenção de aproximadamente 2.800 mortes por ano.

Nos Estados Unidos, Smith et al. ${ }^{46}$, com o intuito de avaliar o progresso do tratamento do câncer, analisaram as tendências de mortalidade por câncer em crianças e adolescentes com idade $<20$ anos no período entre 1975 2010. Nessa análise, observou-se que a mortalidade por câncer nessa população apresentou declínio significativo no período entre 2002-2010 (APC: -2,4\% [IC 95\%: -3,2;-1,7]), sendo similar ao observado em 1975 1998 (APC -2,7\% [IC 95\%: -2,8;-2,5]). Declínios significativos na mortalidade foram observados em todos os grupos etários ( $<20$ anos, 15-19 anos e $<15$ anos). Para o grupo com idade $<20$ anos, foi observado declínio significativo para LLA (APC: -3,1), LMA (APC: -2,3), LNH (APC: -4,40), LH (APC: -7,6), ganglioneuroblastoma (APC: -1,9), cânceres do SNC (APC: -1,1) e cânceres gonodais (APC: -6,1). Além disso, no período de 2000-2010, foram observadas, para o grupo etário de 15-19 anos, taxas de mortalidade iguais ou maiores ao grupo com idade $<15$ anos.

Ressalte-se que, no resultado original dos estudos de Ferlay et al. ${ }^{6}$, Bosetti et al..$^{44}$ e Chatenoud et al. ${ }^{45}$, as taxas estão apresentadas por $100 \mathrm{mil}$; mas, para efeito de comparação, optou-se por convertê-las para milhão.

\section{MORTALIDADE POR CÂNCER EM CRIANÇAS E ADOLESCENTES NO BRASIL}

No Brasil, Ferman et al. ${ }^{47}$ analisaram a mortalidade do câncer infantil (0-14 anos) em cinco regióes geográficas brasileiras ao longo de três décadas. Nessa análise, foi observado que, de um modo geral, as taxas de mortalidade ajustadas por idade apresentaram uma tendência para a estabilidade em todo o país (36,91 mortes por milhão em 1979 e 39,83 mortes por milhão em 2008), com diminuição das taxas de mortalidade de câncer infantil nas Regiōes Sul e Sudeste (1,2 a 1,6\% ao ano, respectivamente), estabilidade na Região Centro-Oeste, e aumento nas Regióes Norte e Nordeste. A mortalidade de todos os cânceres infantis de 1979-2008 demonstrou um discreto, mas significativo declínio (aproximadamente $0,5 \%$ ao ano) em meninos (variação percentual média anual, AAPC: -0,34), sendo estável em meninas (AAPC: $-0,03)$. Quando analisado os tumores separadamente, a mortalidade por leucemia diminuiu significativamente durante todo o período (14,33 mortes por milhão em 1979 e 13,83 mortes por milhão em 2008).

Outro estudo brasileiro analisou as tendências na mortalidade por câncer entre adolescentes e adultos jovens com idade entre 15-29 anos, no período de 1979-2013. Nesse estudo, foram observadas taxas de mortalidade de 54 mortes por milhão entre adolescentes e adultos jovens com idade entre 15-19 anos, 61 mortes por milhão entre 20-24 anos e 88 mortes por milhão entre 25-29 anos. Leucemias, linfomas e tumores do SNC apresentaram as maiores taxas em todos os grupos etários. As taxas de câncer do colo do útero foram mais altas entre 25-29 anos. Houve aumento significativo nas tendências de mortalidade nas Regióes Norte e Nordeste para todos os grupos de tumores, especialmente tumores do SNC. Uma pequena diminuição na taxa de mortalidade por linfomas foi observada nas Regiôes Sul e Sudeste ${ }^{48}$.

Além disso, o Instituto Nacional de Câncer José Alencar Gomes da Silva (INCA) ${ }^{40}$, em sua mais recente publicação sobre mortalidade do câncer em crianças, adolescentes e adultos jovens no Brasil, observou que os óbitos por câncer ocuparam a segunda causa de morte em crianças, adolescentes e adultos jovens (0-29 anos). No período de 2009-2013, a taxa média de mortalidade ajustada por idade observada foi de 32,07 por milhão na faixa etária de 0-14 anos, 44,22 por milhão na faixa etária de 0-19 anos, 54,01 por milhão na faixa etária de 15-19 anos, e 66,97 por milhão na faixa etária de 15-29 anos. Entre as principais causas de morte por câncer em crianças com idade entre 0-14 anos, as leucemias foram o tipo de câncer de maior mortalidade (14,94 mortes por milhão) seguido pelos tumores do $\mathrm{SNC}(10,26$ mortes por milhão) 
e LNH (2,70 mortes por milhão) em ambos os sexos. Em adolescentes e adultos jovens (15-29 anos), manteve-se o padráo, com as leucemias como o tipo de câncer de maior mortalidade (13,96 mortes por milhão), seguido pelos tumores do SNC (9,56 mortes por milhão) e LNH (5,14 mortes por milhăo), sendo destaque para o câncer do colo do útero como principal causa de mortalidade no sexo feminino na faixa etária de 25-29 anos.

\section{CONCLUSÃO}

Esta revisão descreve os resultados de análises realizadas nesta última década em relaçáo à incidência, mortalidade e tendências do câncer em crianças e adolescentes, fornecendo informaçóes atualizadas sobre o panorama geral e comportamento da doença em diversas regióes geográficas. As informaçóes dos registros de câncer são, portanto, indispensáveis no enfrentamento do câncer na população pediátrica, principalmente nos países em desenvolvimento, onde o impacto do câncer é pouco conhecido, ao passo que seu efeito sobre a populaçáo está aumentando. A elucidação da extensão do câncer nessa população permite que programas de controle de câncer sejam planejados com eficiência, não apenas implementando estratégias de prevenção, mas também padróes de cuidado por meio de melhorias na adoção de estratégias de tratamento integrado para melhorar as taxas de mortalidade por câncer em crianças e adolescentes no Brasil e no mundo.

\section{CONTRIBUIÇÕES}

Todas as autoras contribuíram em todas as etapas do manuscrito.

\section{DECLARAÇÃO DE CONFLITO DE INTERESSES}

Nada a declarar.

\section{FONTES DE FINANCIAMENTO}

Não há.

\section{REFERÊNCIAS}

1. Steliarova-Foucher E, Stiller C, Kaatsch P, Berrino F, Coebergh JW, Lacour B. et al. Geographical patterns and time trends of cancer incidence and survival among children and adolescents in Europe since the 1970s (the ACCIS project): an epidemiological study. Lancet. 2004;364(9451):2097-2105. Cited in: PubMed; PMID: 15589307.
2. Boyle P, Levin B, editors. World cancer report 2008. Lyon, France: International Agency for Research on Cancer; 2008.

3. American Cancer Society. Cancer facts \& figures 2014. Atlanta: American Cancer Society; 2014.

4. Parkin DM, Stiller CA, Draper GJ, Bieber CA. The international incidence of childhood cancer. Int J Cancer. 1988,42(4):511-520. Cited in: PubMed; PMID: 3170025.

5. Buka I, Koranteng S, Vargas AR. Trends in childhood cancer incidence: review of environmental linkages. Pediatr Clin North Am. 2007,54(1):177-203.

6. Ferlay J, Soerjomataram I, Ervik M, Dikshit R, Eser S, Mathers C, et al., editors. GLOBOCAN 2012: estimated cancer incidence and prevalence worldwide in 2012 v1.0. Lyon, France: International Agency for Research on Cancer; 2013. (IARC CancerBase; no. 11).

7. Stewart BW; Wild PW, editors. World Cancer Report 2014. Lyon, France: International Agency for Research on Cancer; 2014.

8. Little J. Introduction. In: Little J. Epidemiology of childhood cancer. Lyon, France: IARC; c1999. P.1-9. (IARC Scientific Publications; 149).

9. Stiller CA. International patterns of cancer incidence in adolescents. Cancer Treat Rev. 2007;33(7):631-645. Cited in: PubMed; PMID: 17329031.

10. Linabery AM, Ross JA. Trends in childhood cancer incidence in the U.S. (1992-2004). Cancer. 2008, 112(2):416-432. Cited in: PubMed; PMID: 18074355.

11. Matthes R, Ziegelberger G, editors. Risk factors for childhood leukaemia. Radiation Protection Dosimetry. 2008;132(2 Spec No):107-274.

12. Valsecchi MG, Steliarova-Foucher E. Cancer registration in developing countries: luxury or necessity? Lancet Oncol. 2008;9(2):159-167. Cited in: PubMed; PMID: 18237850 .

13. Greaves M. Infection, immune responses and the aetiology of childhood leukaemia. Nat Rev Cancer. 2006;6(3):193-203. Cited in: PubMed; PMID: 16467884 .

14. Steliarova-Foucher E, Stiller C, Lacour B, Kaatsch P. International classification of childhood cancer, third edition. Cancer. 2005;103(7):1457-1467. Cited in: PubMed; PMID: 15712273.

15. Birch JM, Alston RD, Kelsey AM, Quinn MJ, Babb P, McNally RJ. Classification and incidence of cancers in adolescents and young adults in England 1979-1997. Br J Cancer. 2002;87(11):1267-1274. Cited in: PubMed; PMID: 12439716.

16. Stiller CA, Desandes E, Danon SE, Izarzugaza I, Ratiu A, Vassileva-Valerianova Z, et al. Cancer incidence and survival in European adolescents (1978-1997). Report from the automated childhood cancer information 
system project. Eur J Cancer. 2006;42(13):2006-2018. Cited in: PubMed; PMID: 16919767.

17. Baade PD, Youlden DR, Valery PC, Hassall T, Ward L, Green AC, et al. Trends in incidence of childhood cancer in Australia, 1983-2006. Br J Cancer. 2010; 102(3):620-626. Cited in: PubMed; PMID: 20051948.

18. Baba S, Ioka A, Tsukuma H, Noda H, Ajiki W, Iso $\mathrm{H}$. Incidence and survival trends for childhood cancer in Osaka, Japan, 1973-2001. Cancer Sci. 2010;101(3):787-792. Cited in: PubMed; PMID: 20132215.

19. Kohler BA, Ward E, McCarthy BJ, Schymura MJ, Ries LA, Eheman C, et al. Annual report to the nation on the status of cancer, 1975-2007, featuring tumors of the brain and other nervous system. J Natl Cancer Inst. 2011;103(9):714-736. Cited in: PubMed; PMID: 21454908.

20. Parkin DM, Ferlay J, Hamdi-Chérif M, Sitas F, Thomas JO, Wabinga $\mathrm{H}$, et al., editors. Cancer in Africa: epidemiology and prevention. Lyon, France: IARC Press; 2003. Chapter 5, Childhood cancer; p. 381-396. (IARC Scientific Publications; 153).

21. Fajardo-Gutiérrez A, Juárez-Ocaña $S$, GonzálezMiranda G, Palma-Padilla V, Carreón-Cruz R, OrtegaAlvárez $\mathrm{MC}$, et al. Incidence of cancer in children residing in ten jurisdictions of the Mexican Republic: importance of the Cancer registry (a population-based study). BMC Cancer. 2007;7:68. Cited in: PubMed; PMID: 17445267.

22. Swaminathan R, Rama R, Shanta V. Childhood cancers in Chennai, India, 1990-2001: incidence and survival. Int J Cancer. 2008;122(11):2607-2611. Cited in: PubMed; PMID: 18324630.

23. Bao PP, Zheng Y, Wang CF, Gu K, Jin F, Lu W. Time trends and characteristics of childhood cancer among children age 0-14 in Shanghai. Pediatr Blood Cancer. 2009;53(1):13-61. Cited in: PubMed; PMID: 19260104.

24. Lacour B, Guyot-Goubin A, Guissou S, Bellec S, Désandes E, Clavel J. Incidence of childhood cancer in France: National Children Cancer Registries, 2000-2004. Eur J Cancer Prev. 2010;19(3):173-181. Cited in: PubMed; PMID: 20361423.

25. Moradi A, Semnani S, Roshandel G, Mirbehbehani $\mathrm{N}$, Keshtkar A, Aarabi M, et al. Incidence of childhood cancers in Golestan province of Iran. Iran J Pediatr.2010;20(3):335-342. Cited in: PubMed; PMID:23056726.

26. Wiangnon S, Veerakul G, Nuchprayoon I, Seksarn P, Hongeng S, Krutvecho T, et al. Childhood cancer incidence and survival 2003-2005, Thailand: study from the Thai Pediatric Oncology Group. Asian Pac J Cancer Prev. 2011;12(9):2215-2220. Cited in: PubMed; PMID: 22296359.
27. Kaatsch P, Spix C, editors. German Childhood Cancer Registry. Annual Report 2011. Mainz (Germany): Institute of Medical Biostatistics, Epidemiology and Informatics (IMBEI) at the University Medical Center of the Johannes-Gutenberg University Mainz; 2012.

28. Howlader N, Noone AM, Krapcho M, Garshell J, Neyman N, Altekruse SF, et al., editors. SEER Cancer Statistics Review, 1975-2010. [Internet]. Bethesda, MD: National Cancer Institute; 2013. [Acessado em: 20 abril de 2018]. Available from: https://seer.cancer. gov/csr/1975_2010/.

29. Moreno F, Loria D, Abriata G, Terracini B; ROHA network. Childhood cancer: incidence and early deaths in Argentina, 2000-2008. Eur J Cancer. 2013;49(2):465-473. Cited in: PubMed; PMID: 22980725.

30. Magrath I, Steliarova-Foucher E, Epelman S, Ribeiro RC, Harif M, Li CK, et al. Paediatric cancer in lowincome and middle-income countries. Lancet Oncol. 2013;14(3):104-116. Cited in: PubMed; PMID: 23434340 .

31. Amir H, Kaaya EE, Manji KP, Kwesigabo G, Biberfeld P. Kaposi's sarcoma before and during a human immunodeficiency virus epidemic in Tanzanian children. Pediatr Infect Dis J. 2001;20(5):518-521. Cited in: PubMed; PMID: 11368110.

32. Aka P, Kawira E, Masalu N, Emmanuel B, Brubaker G, Magatti J, et al. Incidence and trends in Burkitt lymphoma in northern Tanzania from 2000 to 2009. Pediatr Blood Cancer. 2012;59(7):1234-1238. Cited in: PubMed; PMID: 22618958.

33. Ramot B, Magrath I. Hypothesis: the environment is a major determinant of the immunological sub-type of lymphoma and acute lymphoblastic leukaemia in children. Br J Haematol. 1982;50(2):183-89. Cited in: PubMed; PMID: 6977370.

34. Stiller CA, Parkin DM. Geographic and ethnic variations in the incidence of childhood cancer. $\mathrm{Br}$ Med Bull.1996;52(4): 682-703. Cited in: PubMed; PMID: 9039726.

35. Ferlay, F Bray, P Pisani, Parkin DM. editors. GLOBOCAN 2002: cancer incidence, mortality and prevalence worldwide v2.0. Lyon, France: International Agency for Research on Cancer; 2004. (IARC CancerBase; no. 5).

36. Kaatsch P, Steliarova-Foucher E, Crocetti E, Magnani C, Spix C, Zambon P. Time trends of cancer incidence in European children (1978-1997): report from the Automated Childhood Cancer Information System project. Eur J Cancer. 2006;42(13):1961-1971. Cited in: PubMed; PMID: 16919764.

37. Steliarova-Foucher E, Colombet M, Ries LAG, Moreno F, Dolya A, Bray F, et al. International incidence of childhood cancer, 2001-10: a population-based registry 
study. Lancet Oncol. 2017;18(6):719-731. Cited in: PubMed; PMID: 28410997.

38. Siegel DA, King J, Tai E, Buchanan N, Ajani UA, Li J. Cancer incidence rates and trends among children and adolescents in the United States, 2001-2009. Pediatrics. 2014;134(4):e945-955. Cited in: PubMed;PMID: 25201796.

39. Burkhamer J, Kriebel D, Clapp R. The increasing toll of adolescent cancer incidence in the US. PLoS One. 2017;24;12(2):e0172986. Cited in: PubMed; PMID: 28235028 .

40. Instituto Nacional de Câncer José Alencar Gomes da Silva. Incidência, mortalidade e morbidade hospitalar por câncer em crianças, adolescentes e adultos jovens no Brasil: informaçóes dos registros de câncer e do sistema de mortalidade. [Internet]. Rio de Janeiro: INCA; 2016. [Acessado em: 20 abril de 2018]. Disponível em: http://www1.inca.gov.br/wcm/incidencia/2017/pdf/ versao-completa.pdf

41. de Camargo B, Santos MO, Rebelo MS, Reis RS, Ferman S, Noronha CP, et al. Cancer incidence among children and adolescents in Brazil: first report of 14 population-based cancer registries. Int J Cancer. 2010; 126(3): 715-720. Cited in: PubMed; PMID: 19642142.

42. de Camargo B, Ferreira JM, Reis RS, Ferman S, Santos MO, Pombo-de-Oliveira MS. Socioeconomic status and the incidence of non-central nervous system childhood embryonic tumors in Brazil. BMC Cancer. 2011;11:160. Cited in: PubMed; PMID: 21545722.

43. Ferreira JM, Klumb CE, Reis RS, Santos MO, Oliveira JF, de Camargo B, et al. Lymphoma subtype incidence rates in children and adolescents: first report from Brazil. Cancer Epidemiol. 2012; 36(4):e221-e226. Cited in: PubMed; PMID: 2552334.

44. Bosetti C, Bertuccio P, Chatenoud L, Negri E, Levi F, La Vecchia C. Childhood cancer mortality in Europe, 1970-2007. Eur J Cancer. 2010; 46(2):384-394. Cited in: PubMed; PMID: 19818600.

45. Chatenoud L, Bertuccio P, Bosetti C, Levi F, Negri E, La Vecchia C. Childhood cancer mortality in America, Asia, and Oceania, 1970 through 2007. Cancer. 2010. 116(21):5063-5074. Cited in: PubMed; PMID: 20629033.

46. Smith MA, Altekruse SF, Adamson PC, Reaman GH, Seibel NL. Declining childhood and adolescent cancer mortality. Cancer. 2014;120(16):2497-2506. Cited in: PubMed; PMID: 24853691.

47. Ferman S, Santos MO, Ferreira JM, Reis RS, Oliveira JF, Pombo-de-Oliveira MS, et al. Childhood cancer mortality trends in Brazil, 1979-2008. Clinics (São Paulo). 2013;68(2):219-224. Cited in: PubMed; PMID: 23525319.

48. Balmant NV, de Souza Reis R2, de Oliveira Santos M2, Pinto Oliveira J2, de Camargo B. Trends in cancer mortality among adolescents and young adults in Brazil. J Adolesc Young Adult Oncol. 2017;6(2):341-347. Cited in: PubMed; PMID: 28051344. 